\title{
New Mutations of the ID1 Gene in Acute Myeloid Leukemia Patients
}

\author{
Chintana Tochareontanaphol ${ }^{a}$ Thivaratana Sinthuwiwat ${ }^{a}$ Borisuit Buathong ${ }^{b}$ \\ Thunyatip Thita $^{b}$ Somying Promso ${ }^{c}$ Saowakon Paca-uccaralertkun ${ }^{b}$ \\ ${ }^{a}$ Cancer Cytogenetic Unit, Chulabhorn Hospital, Bangkok, b Department of Microbiology, Faculty of Science, \\ Mahidol University, and ' Virology and Molecular Microbiology Unit, Department of Pathology, Ramathibodi \\ Hospital, Mahidol University, Bangkok, Thailand
}

\section{Key Words}

Inhibitor of DNA binding 1 protein - Mutation .

Acute myeloid leukemia

\begin{abstract}
Objectives: Overexpression of the inhibitor of DNA binding 1 (ID1) protein is found in many types of cancer. In acute myeloid leukemia (AML), the expression of ID1 is induced by abnormal tyrosine kinases, such as FLT3 and BCR-ABL. High level expression of ID1 is associated with poor prognosis in young patients. We aimed to explore the ID1 mutation and its prognosis in AML patients. Methods: Two hundred and sixty-three AML patients were included. Cytogenetic results and ID1 mutation were compared. The ID1 gene was amplified by nested PCR, and the mutation was identified by direct sequencing. Results: Four new ID1 mutations (G40C, A124G, A230G, A349G) were identified in the normal karyotype patients. The A349G mutation, located in the nuclear export signal domain of the ID1 protein, was predicted by the in silico method as a damaged protein. Meanwhile, another new mutation, A290G, found in cases with 11q23 deletion, corresponded to the amino acid 97 in the helix 1 position of the ID1 protein. It could interfere with the dimerization of ID1 and EST-1, leading to a disruption of cell proliferation.
\end{abstract}

Conclusions: In this study, we found 5 mutations in $260 \mathrm{AML}$ patients. ID1 mutations were not commonly observed in AML. This may differ in other hematologic malignancies. Further studies in other types of hematologic malignancy will help to clarify the importance of ID1 mutations.

(c) 2015 S. Karger AG, Basel

\section{Introduction}

The inhibitor of DNA binding 1 (ID1) protein belongs to a family of helix-loop-helix proteins consisting of ID1, ID2, ID3 and ID4. Normally, ID proteins function as negative regulators of helix-loop-helix transcription factors [1]. Expression of the ID1 protein enhances cell proliferation, regulates cell survival and inhibits cellular differentiation in certain cell types such as fibroblast [2], epidermis [3] and Sertoli cells [4]. The ID1 gene, located on chromosome 20 at 20q11.21 [5], contains 1,239 bp encoding for 2 exons. In normal adult tissues, the expression level of ID1 can be either absent or low. However, the high expression level of ID1 occurs in a variety of benign and malignant tumors, for example lung cancer [6], breast cancer [7], prostate cancer [8], bladder cancer [9] etc. The increased levels of ID1

\section{KARGER 125}

(c) 2015 S. Karger AG, Base

1015-2008/15/0821-0043\$39.50/0 
expression are correlated with aggressive tumor phenotypes and poor prognosis in non-small cell lung cancer [10] and colorectal cancer [11] and are associated with a shorter overall survival and disease-free survival time in breast cancer [12]. In 2008, Suh et al. [13] demonstrated that the suppression of ID1 expression by small interfering RNA in an in vitro study causes cancer cells to be less invasive and more sensitive to chemotherapeutic drugs. In vivo and in vitro studies of hematopoietic malignancies revealed that ID1 can immortalize myeloid progenitors and promote myeloid proliferation diseases. Additionally, the blocking of the ID1 gene in premature cells leads to the disruption of B-cell development [13]. Also, ID1 is a downstream target of many known oncogenic tyrosine kinases in hematopoietic malignancies such as BCR-ABL, TEL-ABL, FLT3 and PDGFRB [14]. In several previous studies, the important role of ID1 in hematopoietic malignancies was demonstrated [13]. Especially in acute myeloid leukemia (AML), the deletion of the long arm of chromosome 20 is one of the most common chromosome aberrations observed [15]. AML is a heterogeneous neoplasm caused by gene mutations, chromosomal aberrations and deregulation of gene expression. Hence, we aimed to study the mutation of ID1 in AML patients in comparison with cytogenetic results. The incidence of ID1 mutation and its possible protein function were analyzed.

\section{Materials and Methods}

\section{Patients}

A total of 263 AML patients were recruited in this study (fig. 1). They were all diagnosed as AML cases by the morphological study of bone marrow aspirations. Each sample was collected for routine cytogenetic study in our laboratory. Informed consent was obtained from all patients. This study was approved by the Ethics Committee of the Chulabhorn Hospital.

\section{Cytogenetic Study}

The chromosome study was performed on fresh bone marrow specimens by culturing for $24 \mathrm{~h}$ in $20 \%$ FCS-RPMI medium without mitogen stimulation. The chromosomes were stained by the Qbanding method. In each patient, 20 metaphases were analyzed, if possible, and 2 well-banded metaphases were karyotyped. The chromosomal abnormalities were described according to ISCN 2009.

\section{DNA Preparation}

After routine chromosome analysis, the 3:1 methanol acetic acid-fixed cells were stored at $-20^{\circ} \mathrm{C}$. DNA was extracted from these fixed cells using a cell and tissue kit (Gentra systems, Minneapolis, Minn., USA) following the manufacturer's recommended protocol.

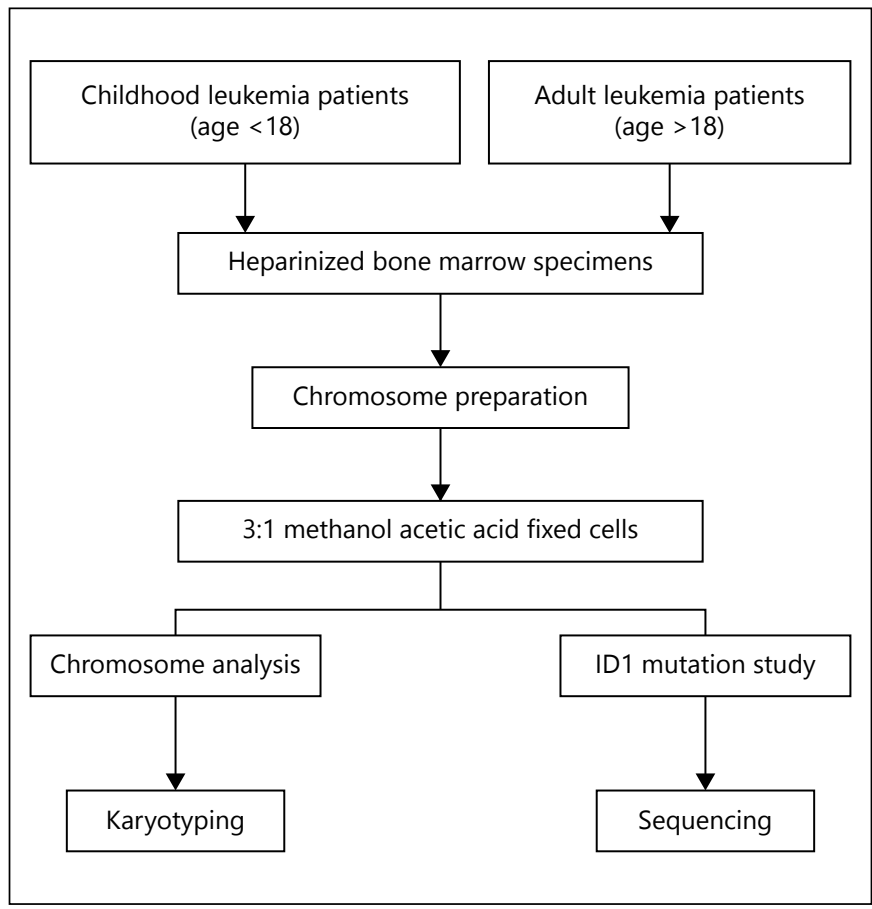

Fig. 1. Flow chart of the classification, chromosome analysis and mutation study of the samples.

\section{Direct Sequencing of the ID1 Exons}

Mutations of the ID1 gene were studied by using the direct sequencing method. The ID1 exons were amplified by nested PCR using 2 sets of primers designed in our laboratory. The sequences of the outer primers were $5^{\prime}$-TCTCATTCCACGTTCT TAACTGTT- $3^{\prime}$ (forward) and $5^{\prime}$-CGCTTTTTCCAGGCTCCT- ${ }^{\prime}$ (reverse). The inner primers were $5^{\prime}$-CGTATCTGCTTCGGG CTTCC-3' (forward) and $5^{\prime}$-CCCCCGCATAGCCAGGTA-3' (reverse). The PCR products were subjected to sequencing by Macrogen Inc., Seoul, Korea. Sequence comparisons and mutation discoveries were carried out using the sequencer software Molecular Evolutionary Genetic Analysis.

\section{Mutation and Functional Assessment}

Mutations identified in this study were mapped onto the human genome using the NCBI Reference Sequence NP_002156.2. The mutations were analyzed using PolyPhen [16] to assess the potential impact of amino acid substitution on protein function. This tool was run using the online service on the PolyPhen website (http://genetics.bwh.harvard.edu/pph/index.html).

\section{Results}

A chromosome analysis was performed in all $263 \mathrm{AML}$ patients. No chromosome results were shown in the 17 patients for whom no metaphases were obtained. The
Tochareontanaphol et al. 
mean age of these patients was 46.89 years ( 120 males, 143 females). The mean age of male and female patients was 48.14 and 49.94 years, respectively. The patterns of chromosome abnormalities are summarized in table 1 . Of these, 28 patients ( 17 females, 11 males) were under 18 years of age. The mean age of pediatric AML was 10.69 years in females and 13.91 years in males. Chromosome abnormalities in pediatric AML were found in $47.06 \%$ $(8 / 17)$ of female patients and $63.64 \%$ (7/11) of male patients. In adult AML, the abnormal chromosomes were found in $47.62 \%(60 / 126)$ of female patients and $39.45 \%$ $(43 / 109)$ of male patients.

The expected PCR products, $617 \mathrm{bp}$, were successfully detected for all 263 patients. In total, ID1 mutations were identified in $2.66 \%$ (7/263) of the samples. Two of them were silent mutations which have been previously reported (http://www.ncbi.nlm.nih.gov/SNP/). Five new mutations were identified (table 2). When the mutations were found, resequencing of the same specimen with both forward and reverse direction was repeated. The new mutations were found in 4 patients with normal chromosome patterns. Only 1 mutation was found in 1 patient with 11q23 deletion (ID: 467). The 5 new mutations (G40C, A124G, A230G, A290G, A349G) were mapped onto the human genome NCBI Reference Sequence NP_002156.2 database dpSNP (HG19). All of these mutations were located in ID1 gene coding regions, and all of them were nonsynonymous mutations, identified as new mutations of the ID1 gene. Furthermore, we analyzed the novel mutations using the PolyPhen software for functional prediction. The software prediction showed that 4 mutations (G40C, A124G, A230G, A290G) are likely to affect the protein function. Only 1 mutation (A349G) has a potential benign activity, as shown in table 2 .

\section{Discussion}

The relationship between ID1 expression and progressive cancer development has been shown in several studies. The high levels of ID1 expression are related to an aggressive phenotype and a poor clinical outcome in many types of cancer [17-19]. Previous observations also showed that ID1 overexpression was found in the aggressive type of AML [20].

According to the single nucleotide polymorphism database, only few mutations of the ID1 gene in AML patients have been identified, and therefore we investigated the mutation rate of the ID1 gene in AML pa-
Table 1. Frequency of common chromosome aberrations in the present study

\begin{tabular}{llc}
\hline Chromosome abnormalities $^{1}$ & Males & Females \\
\hline Monosomy 7 & 5 & 3 \\
Trisomy 8 & 8 & 12 \\
Trisomy 21 & 3 & 9 \\
$t(8 ; 21)(\mathrm{q} 22 ; \mathrm{q} 22)$ & 8 & 5 \\
$\mathrm{t}(9 ; 22)(\mathrm{q} 34 ; \mathrm{q} 11.2)$ & 2 & 5 \\
Rearrangement of 11q23 & 3 & 11 \\
$\mathrm{t}(15 ; 17)(\mathrm{q} 24 ; \mathrm{q} 21)$ & 3 & 9 \\
Inv $(16)(\mathrm{p} 12 \mathrm{q} 13)$ & 2 & 2 \\
\hline
\end{tabular}

${ }^{1}$ Sole chromosome abnormalities.

tients. In our study, mutations of IDI in AML patients were found in $2.66 \%$ of the samples; 1 of them was with 11q23 deletion. That patient's karyotype was 46,XX,del(11)(q23). Most of the ID1 mutations were observed in patients with normal karyotype. Five of the 6 mutations were identified as new mutations. Four of these new mutations were nonsynonymous, and they were predicted by a known algorithm to likely alter the protein function. The mutations of the amino acid positions 77 and 97 are located in the helix 1 area of the ID1 protein [21], while the amino acid position 117 is within the nuclear export signal motif [22]. A study of the ID1 interaction using the in silico method showed that the $\mathrm{H} 97$ has a strong interaction with the K381 of ETS1 [23], a member of the E26 protein family. The dimerization of ID1 and ETS-1 shuts down the CDKN2A (p16INK4a) expression. Under normal circumstances, ETS-1 binds to the promoter region of CDKN2A and induces apoptosis. As shown in ovarian cancer, the suppression of CDKN2A expression promotes abnormal cell proliferation [24]. The peptide aptamer ID1/3-PA7 was designed to inhibit the binding of ID1 and ETS-1 and allows the transcription of CDKN2A [23], triggering cell cycle arrest and apoptosis of malignant cells. The characterization of IDI mutations may provide more insight into drug interactions in the treatment of cancer [25].

From the above data, we hypothesized that the upregulated ID1 gene expression may represent a secondary mechanism to the stimulation of the fusion gene commonly found in AML. Other possible mechanisms may include the mutation of the ID1 gene itself that could alter the ID1 protein function. So, the ID1 gene mutation should be more commonly found in patients 
Table 2. Position and type of mutation in the ID1 gene identified in this study

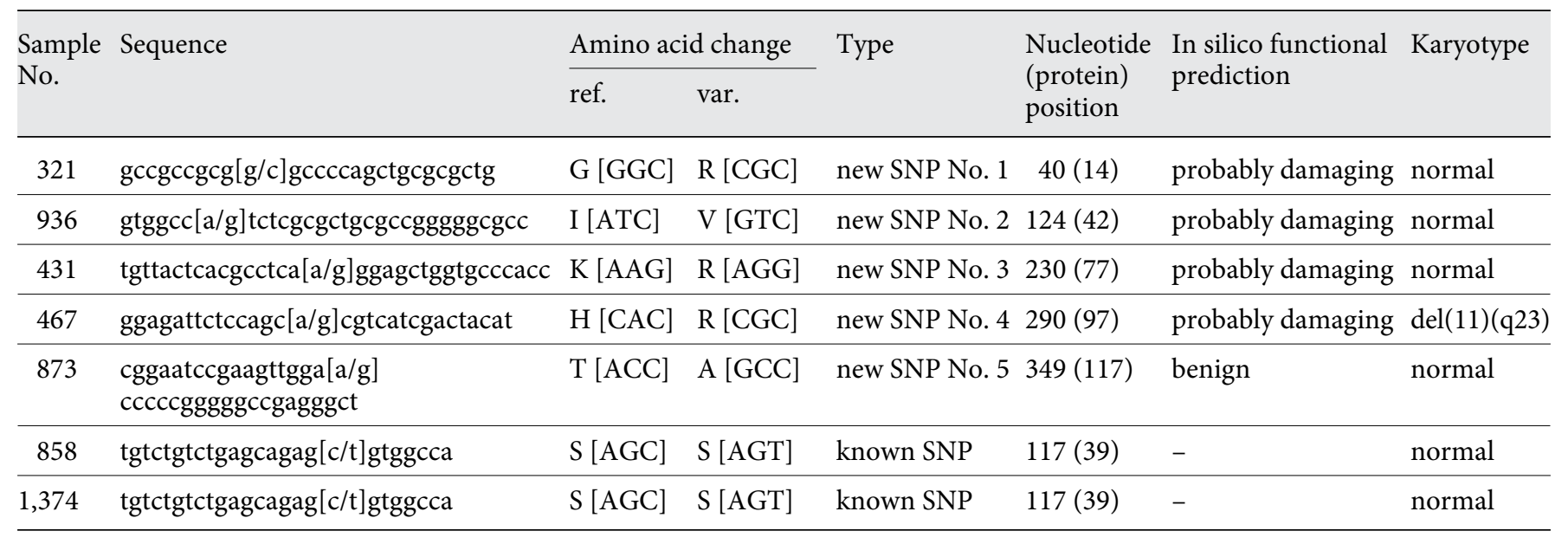

with a normal karyotype. This could be confirmed by using experimental functional assays to determine the effect of each mutation. The high expression level of ID1 may not be an early event in the tumorigenesis pathway.

Nevertheless, ID1 mutations may not be considered common in AML patients. Most of the ID1 mutations in this study were found in patients with a normal karyotype. The ID1 mutation is rare in AML patients, but this may differ in other hematologic malignancies, including acute lymphocytic leukemia, multiple myelomas and myelodysplasia, and needs to be further clarified.

\section{Acknowledgments}

We thank Dr. Fabien Loison for the English editing of this paper.

\section{References}

1 Kadesch T: Consequences of heteromeric interactions among helix-loop-helix proteins. Cell Growth Differ 1993;4:49-55.

$\checkmark 2$ Chambers RC, Leoni P, Kaminski N, Laurent GJ, Heller RA: Global expression profiling of fibroblast responses to transforming growth factor-betal reveals the induction of inhibitor of differentiation- 1 and provides evidence of smooth muscle cell phenotypic switching. Am J Pathol 2003; 162:533-546.

3 Rotzer D, Krampert M, Sulyok S, et al: Id proteins: novel targets of activin action, which regulate epidermal homeostasis. Oncogene 2006;25:2070-2081.

4 Chaudhary J, Sadler-Riggleman I, Ague JM, Skinner MK: The helix-loop-helix inhibitor of differentiation (ID) proteins induce post-mitotic terminally differentiated Sertoli cells to re-enter the cell cycle and proliferate. Biol Reprod 2005;72:1205-1217.

5 The GenCards Human Gene Database. Inhibitor of DNA binding 1, dominant negative helix-loop-helix protein. 2012. http://www. genecards.org/ (accessed August 14, 2012).
6 Pillai S, Rizwani W, Li X, et al: ID1 facilitates the growth and metastasis of non-small cell lung cancer in response to nicotinic acetylcholine receptor and epidermal growth factor receptor signaling. Mol Cell Biol 2011;31: 3052-3067.

-7 McAllister SD, Christian RT, Horowitz MP, et al: Cannabidiol as a novel inhibitor of Id-1 gene expression in aggressive breast cancer cells. Mol Cancer Ther 2007;6:29212927.

8 Ling MT, Lau TC, Zhou C, et al: Overexpression of Id-1 in prostate cancer cells promotes angiogenesis through the activation of vascular endothelial growth factor (VEGF). Carcinogenesis 2005;26:1668-1676.

$>9 \mathrm{Hu} \mathrm{H}$, Wang YL, Wang GW, et al: A novel role of Id-1 in regulation of epithelial-to-mesenchymal transition in bladder cancer. Urol Oncol 2013;31:1242-1253.

10 Kamalian L, Gosney JR, Forootan SS, et al: Increased expression of Id family proteins in small cell lung cancer and its prognostic significance. Clin Cancer Res 2008;14:23182325.
11 Zhao ZR, Zhang ZY, Zhang H, et al: Overexpression of Id-1 protein is a marker in colorectal cancer progression. Oncol Rep 2008; 19: 419-424.

12 Fong S, Itahana Y, Sumida T, et al: Id-1 as a molecular target in therapy for breast cancer cell invasion and metastasis. Proc Natl Acad Sci U S A 2003;100:13543-13548.

$\checkmark 13$ Suh HC, Leeanansaksiri W, Ji M, et al: Id1 immortalizes hematopoietic progenitors in vitro and promotes a myeloproliferative disease in vivo. Oncogene 2008;27:56125623.

14 Tam WF, Gu TL, Chen J, et al: Id1 is a common downstream target of oncogenic tyrosine kinases in leukemic cells. Blood 2008;112: 1981-1992.

15 Kurtin PJ, Dewald GW, Shields DJ, et al: Hematologic disorders associated with deletions of chromosome 20q: a clinicopathologic study of 107 patients. Am J Clin Pathol 1996; 106:680-688

16 Ramensky V, Bork P, Sunyaev S: Human nonsynonymous SNPs: server and survey. Nucleic Acids Res 2003;30:3894-3900. 
17 Schindl M, Oberhuber G, Obermair A, et al: Overexpression of Id-1 protein is a marker for unfavorable prognosis in early-stage cervical cancer. Cancer Res 2001;61:5703-5706.

18 Schindl M, Schoppmann SF, Ströbel T, et al: Level of Id-1 protein expression correlates with poor differentiation, enhanced malignant potential, and more aggressive clinical behavior of epithelial ovarian tumors. Clin Cancer Res 2003;9:779-785.

19 Schoppmann SF, Schindl M, Bayer G, et al: Overexpression of Id-1 is associated with poor clinical outcome in node negative breast cancer. Int J Cancer 2003;104:677-682.
20 Tang R, Hirsch P, Fava F, et al: High Id1 expression is associated with poor prognosis in 237 patients with acute myeloid leukemia. Blood 2009;114:2993-3000.

21 Kiewitz SD, Cabrele C: Synthesis and conformational properties of protein fragments based on the Id family of DNA-binding and cell-differentiation inhibitors. Biopolymers 2005;80:762-774.

22 Muthu K, Panneerselvam M, Jayaraman M, et al: Structural insights into interacting mechanism of ID1 protein with an antagonist ID1/3-PA7 and agonist ETS-1 in treatment of ovarian cancer: molecular docking and dynamics studies. J Mol Model 2012;18: 4865-4884.
23 Ohtani N, Zebedee Z, Huot TJ, et al: Opposing effects of Ets and Id proteins on CDKN2A expression during cellular senescence. Nature 2001;409:1067-1070.

24 Mern DS, Hasskarl J, Burwinkel B: Inhibition of Id proteins by a peptide aptamer induces cell-cycle arrest and apoptosis in ovarian cancer cells. Br J Cancer 2010;103:1237-1244.

25 Li B, Tsao SW, Chan KW, et al: Id1-induced IGF-II and its autocrine/endocrine promotion of esophageal cancer progression and chemoresistance - implications for IGF-II and IGF-IR-targeted therapy. Clin Cancer Res 2014;20:2651-2662. 